\title{
Interval Appendectomy for Plastron Appendicitis 3 Months After Abscess Drainage, Two Case Reports
}

\author{
Omer Engin ${ }^{1}$, Oguzhan Sunamak ${ }^{2}$ \\ ${ }^{1}$ MD, General Surgeon, Buca Seyfi Demirsoy State Hospital, Surgery Department, Izmir, Turkey \\ ${ }^{2}$ MD, General Surgeon, Chief Assistant, Haydarpasa Numune Training and Research Hospital, \\ Surgery Department, Istanbul, Turkey
}

\begin{abstract}
Most of surgeons prefer open interval appendectomy instead of laparoscopic appendectomy after 3 months diagnosed plastron appendicitis. We would like to present that laparoscopic interval appendectomy may be performed easily but this procedure has some problems. Two cases are presented as case reports. Two cases was operated as acute appendicitis and diagnosed plastron appendicitis intraoperatively. Intraperitoneal free seropurulant fluid was drained, drainage tubes were inserted into duoglas pouch and Mc Burney incisions were closed. After three months they were operated as laparoscopically. We advise that ; in the plastron appendicitis the patients must be performed interval appendectomy as laparoscopically. The procedure has some disadvantage but some numerous techniques may be facilitated such as no classical port insertion, patient's position, open entry for first trocar....
\end{abstract}

Keywords: Plastron appendicitis; acute appendicitis; surgery; laparoscopy; laparoscopic appendectomy.

\section{INTRODUCTION}

Appendectomy is one of the most performed operations in general surgery. It cannot be performed in some delayed cases due to plastron formation and interval appendectomy is performed three months later. Lots of the surgeons prefer to do open surgery for interval operation because of the possible adhesions. Our two cases had undergone operation for appendicitis but appendectomy couldn't have been performed due to plastron and drains had been put into the abdomen and operation had been finished. We performed laparoscopic appendectomy to both patients three months later. We wished to share difficulties of such an operation $(1,2)$.

\section{CASe 1}

37 years old male patient was taken into operation for interval appendectomy. The inraabdominal space was entered via subumbilical open method, a $10 \mathrm{~mm}$ trocar was inserted and pneumoperitoneum was done by using carbon dioxide. One $10 \mathrm{~mm}$ trocar and a $5 \mathrm{~mm}$ one were inserted at right middle quadrant just lateral to the rectus muscle at the level of umbilicus and, at linea alba between umbilicus and the symphisis pubis, respectively. There were dense adhesions between old incision scar and intestines and between cecum and intestines intraabdominally. Fibrous bands and adhesions were dissected by using scissors and ligasure, respectively. As ligasure has a thermal effect around, intestines were cared so that they were not affected. After dissecting adhesions, appendix was seen to extend into the pelvic space. Its mesentery, thus vessels, was ligated by using ligasure and appendectomy was completed. The duration of the operation was 45 minutes.

\section{CASe 2}

43 years-old female patient with smoking history of 20 years was operated laparoscopically. Before operation, the patient was treated for her COPD. Open access method was used and subumbilical 10 $\mathrm{mm}$ trocar was inserted. As massive adhesions were present on the right side of the intraabdominal cavity, $5 \mathrm{~mm}$ trocar couldn't be inserted on this side so it is inserted on left lower quadrant just lateral to the rectus muscle. Another $10 \mathrm{~mm}$ trocar was placed at the line between umbilicus and the symhisis pubis and appendix were found to extend medially and toward the pelvic cavity. Ligasure was used for dissection and appendectomy, similar to the that of the first case and the duration was 55 minutes. 


\section{Discussion}

We preferred subumbilical open access and trocar placement method due to possible adhesions of the previous operations. Having massive adhesions, endoscissors use for cutting avascular fibrous bands were preferred in both of the cases. Intestinal adhesions were separated by blunt dissection first by using endoclenches as the endodissector had a sharp tip. Sharp dissection necessitating adhesions were separated by ligasure use at the position that operation table was deviated to left side so that intestinal loops migrated to the left side of the abdominal cavity by the assistance of endoclench. Ligasure was carefully used to prevent intestinal injury by its thermal effect. In our second case, trocar insertion points were modified according to the situation of intraabdominal adhesions $(3,4,5,6)$.

Generally, open surgery may be preferred for interval appendectomy in plastron appendicitis due to massive adhesions. Laparoscopic operation might be possible if vigorous care and patience were given as in our two cases.. We believe that the advantage of laparoscopic appendectomy should be given to such patients, instead of directly deciding to make an open surgery. We want to impress that modification of trocar entry points according to the situation of adhesions can make the operation easier, instead.

As a result, laparoscopy choice is not given up because of the adhesions. A good preoperative preparation might make laparoscopy easier. Open entry for first trocar should be done. Trocars shouldn't inserted classically but modified on patient basis. It is useful to deviate operation table some degree to the patient's left side.

\section{REFERENCES}

[1] Engin, O., Muratli, A., Ucar, A. D., Tekin, V., Calik, B., \& Tosun, A. (2012). The Importance of Fecaliths in the Aetiology of Acute Appendicitis. Chirurgia,107, 756-760.

[2] Engin, O., Calik, B., Yildirim, M., Coskun, A., \& Coskun, G. A. (2011). Gynecologic pathologies in our appendectomy series and literature review.Journal of the Korean Surgical Society, 80(4), 267-271.

[3] Sakorafas, G. H., Sabanis, D., Lappas, C., Mastoraki, A., Papanikolaou, J., Siristatidis, C., \& Smyrniotis, V. (2012). Interval routine appendectomy following conservative treatment of acute appendicitis: Is it really needed. World journal of gastrointestinal surgery, 4(4), 83.

[4] Cuesta, M. A. (2014). Case on Complications After Conservatively Treated Appendicular Phlegmon. In Case Studies of Postoperative Complications after Digestive Surgery (pp. 307310). Springer International Publishing.

[5] Wei, B., Qi, C. L., Chen, T. F., Zheng, Z. H., Huang, J. L., Hu, B. G., \& Wei, H. B. (2011). Laparoscopic versus open appendectomy for acute appendicitis: a metaanalysis. Surgical endoscopy, 25(4), 1199-1208.

[6] Cong-min, S. H. A., \& Sen, L. I. (2010). Clinical efficacy of laparoscopic appendectomy. Jilin Medical Journal, 26, 003.

[7] Swank, H. A., Eshuis, E. J., van Berge Henegouwen, M. I., \& Bemelman, W. A. (2011). Shortand long-term results of open versus laparoscopic appendectomy. World journal of surgery, 35(6), 1221-1226. 